\title{
(C) OPEN ACCESS \\ Corticosteroids for the prevention of bronchopulmonary dysplasia in preterm infants: a network meta-analysis
}

\author{
Linan Zeng, ${ }^{1,2}$ Jinhui Tian, ${ }^{3,4}$ Fujian Song ${ }^{5}$ Wenrui Li, ${ }^{1,6}$ Lucan Jiang, ${ }^{1,6}$ Ge Gui, ${ }^{1,6}$ \\ Yang Zhang, ${ }^{1,6}$ Long $\mathrm{Ge}^{3}{ }^{3}$ Jing Shi, ${ }^{2,7}$ Xin Sun, ${ }^{8}$ Dezhi Mu, ${ }^{2,7}$ Lingli Zhang ${ }^{1,2}$
}

\begin{abstract}
- Additional material is published online only. To view please visit the journal online (http://dx.doi.org/10.1136/ archdischild-2017-313759).
\end{abstract}

For numbered affiliations see end of article.

\section{Correspondence to} Professor Lingli Zhang, Department of Pharmacy, West China Second University Hospital, Sichuan University, Chengdu, China; zhanglingli@ scu.edu.cn

Received 23 July 2017 Revised 20 December 2017 Accepted 21 December 2017 Published Online First 23 February 2018

\section{Linked}

- http://dx.doi.org/10.1136/ fetalneonatal-2018-314842

Check for updates

To cite: Zeng L, Tian J, Song F, et al. Arch Dis Child Fetal Neonatal Ed

2018;103:F506-F511.

\section{ABSTRACT}

Objective To determine the comparative efficacy and safety of corticosteroids in the prevention of bronchopulmonary dysplasia (BPD) in preterm infants. Study design We systematically searched PubMed, EMBASE and the Cochrane Library. Two reviewers independently selected randomised controlled trials (RCTs) of postnatal corticosteroids in preterm infants. A Bayesian network meta-analysis and subgroup analyses were performed.

Results We included 47 RCTs with 6747 participants. The use of dexamethasone at either high dose or low dose decreased the risk of BPD (OR 0.29, 95\% credible interval (Crl) 0.14 to 0.52 ; OR $0.58,95 \% \mathrm{Crl} 0.39$ to 0.76 , respectively). High-dose dexamethasone was more effective than hydrocortisone, beclomethasone and low-dose dexamethasone. Early and long-term dexamethasone at either high dose or low dose decreased the risk of BPD (OR $0.11,95 \% \mathrm{Crl} 0.02$ to $0.4 ; \mathrm{OR} 0.37,95 \% \mathrm{Crl} 0.16$ to 0.67 , respectively). There were no statistically significant differences in the risk of cerebral palsy (CP) between different corticosteroids. However, high-dose and long-term dexamethasone ranked lower than placebo and other regimens in terms of CP. Subgroup analyses indicated budesonide was associated with a decreased risk of BPD in extremely preterm and extremely low birthweight infants (OR 0.60, 95\% Crl 0.36 to 0.93 ).

Conclusions Dexamethasone can reduce the risk of BPD in preterm infants. Of the different dexamethasone regimens, aggressive initiation seems beneficial, while a combination of high-dose and long-term use should be avoided because of the possible adverse neurodevelopmental outcome. Dexamethasone and inhaled corticosteroids need to be further evaluated in large-scale RCTs with long-term follow-ups.

\section{INTRODUCTION}

Bronchopulmonary dysplasia (BPD) is the most common serious pulmonary disease in premature infants. ${ }^{1}$ Approximately $20 \%$ of infants born at $<30$ weeks of gestation or with a weight $<1500$ $\mathrm{g}$ are diagnosed with BPD. In addition, the incidence of BPD appears to be growing in conjunction with the increased survival of infants with low birth weight. ${ }^{2-4}$ Premature infants with BPD are at an increased risk of death, and survivors often have lifelong morbidities, including delayed neurocognitive development. $^{5}$

\section{What is already known on this topic?}

- Systemic postnatal corticosteroids could reduce the risk of bronchopulmonary dysplasia (BPD) in preterm infants.

- Clinicians remain confused as to the corticosteroid to choose, the time to initiate and the dose to give.

\section{What this study adds?}

- For preterm infants with risk of BPD, high-dose dexamethasone appears to be more effective than other corticosteroids.

- The comparison between different dexamethasone regimens supports aggressive initiation but cautions against high-dose and long-term administration because of adverse neurodevelopmental outcome.

- Budesonide is associated with decreased risk of BPD in extremely preterm and extremely low birthweight infants.

Postnatal systemic corticosteroid administration is controversial, mainly because of the concern for its adverse neurological effects. ${ }^{6-12}$ There were conflicting results from studies assessing the effects of diminished corticosteroid administration on the incidence and severity of BPD following the 2002 policy statements of the American Academy of Pediatrics (AAP). ${ }^{13-15}$ Although the AAP revised the statement in 2010, the role of corticosteroids in the management of BPD remains uncertain. ${ }^{16}$ However, clinicians still use corticosteroids in approximately $5 \%-32 \%$ of preterm infants to prevent and treat BPD. ${ }^{17}$ Therefore, identifying the optimal corticosteroid and regimen remains clinically relevant and important. ${ }^{18} 19$

New clinical trials, meta-analyses and follow-up studies have been published since 2010, warranting an updated review of the available information. Due to different comparators used in clinical trials, a pair-wise meta-analysis cannot be used to evaluate comparative effects of different corticosteroids and regimens. Therefore, we conducted a network meta-analysis of all relevant randomised evidence to comprehensively compare and rank the different corticosteroids used to prevent BPD. 


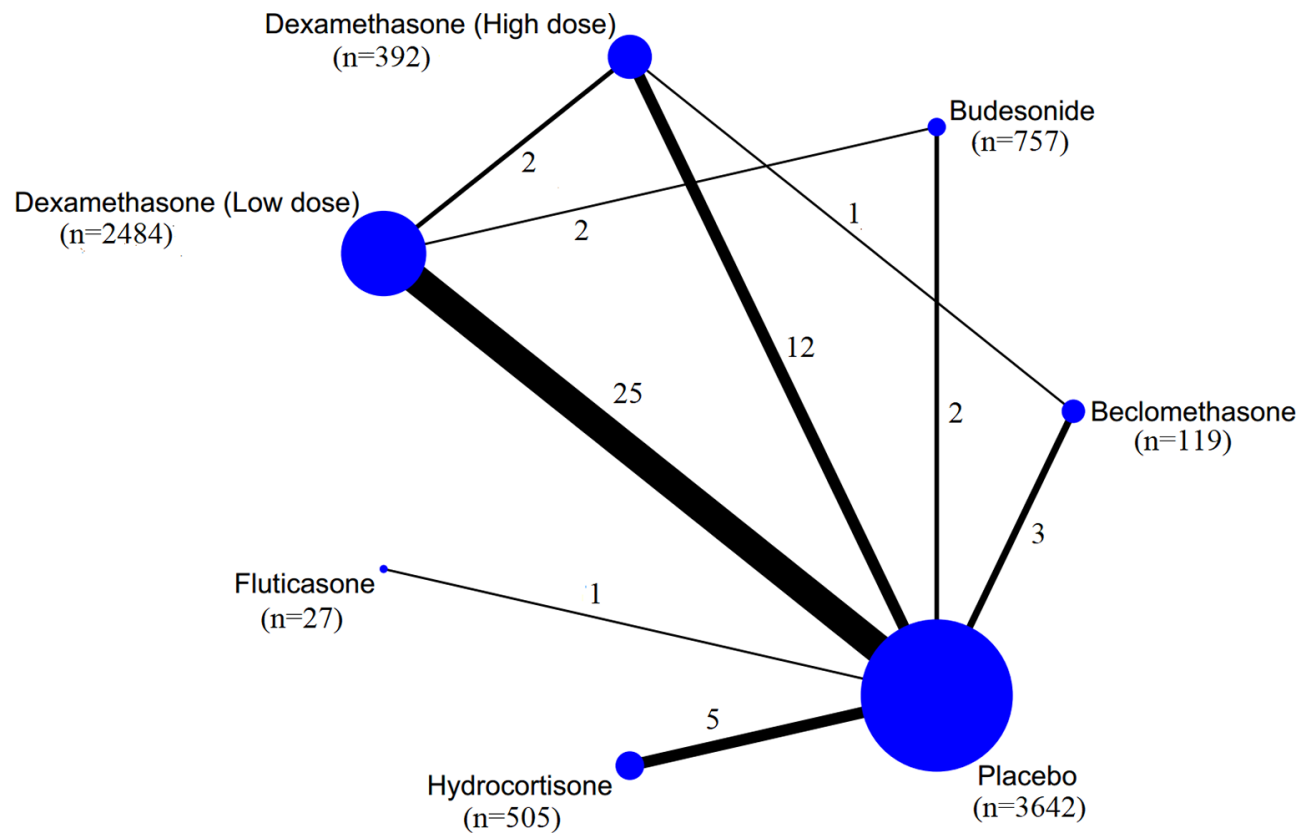

Figure 1 Network of corticosteroids for the prevention of bronchopulmonary dysplasia at 36 weeks' postmenstrual age. The circle size reflects the number of participants, and the line width reflects the number of direct comparisons. No connecting line between two treatments indicates that there was no direct comparison.

\section{METHODS}

\section{Study search and selection}

We systematically searched PubMed (1966 to June 2016), EMBASE (1974 to June 2016) and the Cochrane Library (Issue 6,2016 ) to identify relevant randomised controlled trials (RCTs) in humans published in English. We also manually searched the reference lists of retrieved trials and systematic reviews (online supplementary appendix 1). ${ }^{20}$ Two reviewers independently assessed the trials for eligibility and included RCTs that evaluated the safety and efficacy of postnatal corticosteroid use for BPD prevention in preterm infants ( $<37$ weeks). Studies that used postnatal surfactant and corticosteroids together were also included. Studies that assessed the combined use of corticosteroids and beta agonists were excluded. ${ }^{21}$

\section{Data extraction and quality assessment}

The primary outcome assessed was BPD at 36 weeks' postmenstrual age (PMA). Secondary outcomes included BPD at 28 days' postnatal age (PNA), death at 36 weeks' PMA and 28 days PNA, and a diagnosis of cerebral palsy (CP) in a long-term follow-up. Two independent reviewers extracted the baseline characteristics, methods and outcomes of the included studies. Patients were categorised by gestational weeks (extremely preterm: $<28$ weeks; preterm: $28-37$ weeks) and by birth weight (extremely low birth weight: $<1000 \mathrm{~g}$; very low birth weight: $1000-1500 \mathrm{~g}$; low birth weight: $1500-2500 \mathrm{~g}$ ). Dexamethasone regimens were categorised by the timing of initiation (early initiation: $\leq 7$ days' PNA; late initiation: $>7$ days' PNA), total dose (low dose: $\leq 3.0 \mathrm{mg} / \mathrm{kg}$; high dose: $>3.0 \mathrm{mg} /$ $\mathrm{kg}$ ) and duration (short term: $\leq 3$ days; long term: $>3$ days). ${ }^{22}$ Intention-to-treat analysis data were used whenever available. We contacted the authors of the included studies for missing data by email. Two independent reviewers assessed the risk of bias in trials using the Cochrane risk of bias tool, with any discrepancies resolved by consensus. ${ }^{20}$

\section{Statistical analysis}

We conducted a Bayesian network meta-analysis using the WinBUGS V.1.4.3 software (MRC Biostatistics Unit, Cambridge, UK) and adopted a random-effects model with vague priors for multiarm trials. ${ }^{2324}$ Pooled estimates and the probability of each treatment being the best were obtained by the Markov chain Monte Carlo method. Model convergence was assessed by trace plots and Brooks-Gelman-Rubin plots. ${ }^{25}$ The results of dichotomous outcomes were reported as posterior medians or ORs with $95 \%$ credible intervals (CrIs). The surface under the cumulative ranking curve (SUCRA) was calculated as a percentage to estimate the probability that an intervention was the best. ${ }^{26}$ Prespecified subgroup analyses were performed according to gestational age and birth weight. ${ }^{27}$ The interactions between the intervention effect and subgroups were assessed using a heterogeneity test in meta-analysis. ${ }^{28} 29$ Network geometry was performed with STATA V.13.0 software, in which the size of the nodes and thickness of the edges were proportional to sample sizes and numbers of trials involved, respectively.

\section{RESULTS}

\section{Literature search}

Of the 557 citations initially identified by searching bibliographical databases, 449 were excluded through title and abstract screening. We examined the full text of 108 reports, and identified 66 publications describing 47 RCTs that were eligible for network meta-analysis (online supplementary appendix 2). We contacted 19 corresponding authors of the included studies with missing data, and received additional data from 6 of the 19 authors (online supplementary appendix 3).

\section{Characteristics of the included studies}

The included trials evaluated five corticosteroids and involved a total of 6747 randomly assigned participants. The sample 
Original article

Table 1 Results of network meta-analysis: relative effects of different corticosteroids versus placebo on BPD and CP

\begin{tabular}{|c|c|c|c|}
\hline \multirow[b]{2}{*}{ Outcomes } & \multirow{2}{*}{$\begin{array}{l}\text { Direct drug comparison studies/participants } \\
\text { (n/N) }\end{array}$} & \multicolumn{2}{|c|}{ OR: median $(95 \% \mathrm{Crl})$} \\
\hline & & Direct evidence & All evidence \\
\hline \multicolumn{4}{|c|}{ Network estimates for different corticosteroids } \\
\hline \multicolumn{4}{|l|}{ BPD at 36 weeks' PMA } \\
\hline Dexamethasone (high dose) & $6 / 659$ & $0.34(0.20$ to 0.57$)$ & $0.29(0.14$ to 0.52$)$ \\
\hline Dexamethasone (low dose) & $13 / 2180$ & 0.66 (0.54 to 0.80$)$ & 0.59 (0.39 to 0.77$)$ \\
\hline Hydrocortisone & $5 / 1022$ & $0.80(0.61$ to 1.05$)$ & 0.74 (0.43 to 1.14$)$ \\
\hline Budesonide & $2 / 886$ & $0.60(0.45$ to 0.81$)$ & 0.58 (0.29 to 1.06$)$ \\
\hline Fluticasone & $1 / 53$ & & 0.32 (0.08 to 1.29$)$ \\
\hline Beclomethasone & $3 / 352$ & 0.78 (0.46 to 1.35$)$ & 0.81 (0.40 to 1.55$)$ \\
\hline \multicolumn{4}{|l|}{ Cerebral palsy } \\
\hline Dexamethasone (high dose) & $5 / 307$ & 2.30 (1.22 to 4.36$)$ & 2.02 (0.60 to 4.67 ) \\
\hline Dexamethasone (low dose) & $3 / 245$ & $0.61(0.28$ to 1.34$)$ & $0.81(0.26$ to 3.13$)$ \\
\hline Hydrocortisone & $3 / 334$ & 1.09 (0.57 to 2.11$)$ & 1.79 (0.64 to 15.96$)$ \\
\hline Beclomethasone & $1 / 56$ & & $1.47(0.12$ to 18.47$)$ \\
\hline \multicolumn{4}{|c|}{ Network estimates for different dexamethasone regimens } \\
\hline \multicolumn{4}{|l|}{ BPD at 36 weeks' PMA } \\
\hline Dexamethasone-LHL & $2 / 148$ & $0.38(0.19$ to 0.76$)$ & 0.33 (0.09 to 1.03$)$ \\
\hline Dexamethasone-LHS & 0 & NR & $0.26(0.02$ to 2.66$)$ \\
\hline Dexamethasone-LLL & $5 / 231$ & 0.63 (0.25 to 1.57$)$ & $0.65(0.22$ to 1.63$)$ \\
\hline Dexamethasone-EHL & $3 / 140$ & $0.12(0.04$ to 0.40$)$ & $0.11(0.02$ to 0.41$)$ \\
\hline Dexamethasone-EHS & $1 / 88$ & 0.91 (0.39 to 2.10$)$ & 0.91 (0.19 to 4.24$)$ \\
\hline Dexamethasone-ELL & 2/106 & $0.56(0.43$ to 0.75$)$ & 0.37 (0.16 to 0.67$)$ \\
\hline Dexamethasone-ELS & $5 / 1022$ & 0.76 (0.56 to 1.02$)$ & 0.77 (0.39 to 1.53$)$ \\
\hline \multicolumn{4}{|l|}{ Cerebral palsy } \\
\hline Dexamethasone-LHL & $2 / 123$ & 2.38 (0.95 to 5.98$)$ & 2.75 (0.88 to 8.01$)$ \\
\hline Dexamethasone-LLL & $1 / 56$ & 0.67 (0.20 to 2.28$)$ & $0.87(0.22$ to 3.70$)$ \\
\hline Dexamethasone-EHL & $1 / 146$ & 2.23 (0.92 to 5.40$)$ & $2.30(0.41$ to 13.22$)$ \\
\hline Dexamethasone-LHS & $1 / 22$ & 0.16 (0.01 to 4.69$)$ & 0.10 (1.66E-04 to 3.56$)$ \\
\hline Dexamethasone-ELL & $1 / 144$ & $0.63(0.24$ to 1.65$)$ & 0.53 (0.14 to 1.93$)$ \\
\hline
\end{tabular}

size of the trials ranged from 23 to 856 participants (median 60). The included studies recruited preterm infants with similar ranges of gestational age and birth weight, including extremely preterm with birth weights $<1000 \mathrm{~g}$ (gestational age: median 26.7, IQR 26.0-27.9; birth weight: median 850, IQR 790-1010). According to data from 15 (31.9\%) of the included trials, the average PNA at study entry ranged from 0.14 to 27 days (median 8, IQR 3.04-15.50). Thirty-eight $(80.9 \%)$ of the 47 trials reported the use of prenatal corticosteroids in mothers, and $36(76.6 \%)$ reported the use of surfactant after birth in newborns (online supplementary appendix 4).

\section{Risk of bias}

In approximately $65 \%$ of the trials, the risk of selection bias (sequence generation and allocation concealment) was determined to be low. Blinding of both participants and outcome assessors occurred in 21 trials (44.7\%). Incomplete data were appropriately tackled for the primary outcome (BPD), with $73.3 \%(33 / 45)$ assessed as having a low risk of bias. Forty-two trials $(89 \%)$ were at a low risk of selective outcome reporting. Ten trials (20\%) were deemed to have a high risk of other biases, for reasons that include early trial termination ${ }^{30-36}$ and unbalanced patient characteristics at baseline (online supplementary appendix 5). ${ }^{37} 38$

\section{Network comparisons of the different corticosteroids}

Efficacy: BPD and death

Networks of eligible comparisons for the prevention of BPD are presented in figure 1 . Nine $(32.1 \%)$ of the 28 available pair-wise comparisons showed direct evidence supporting BPD prevention at 36 weeks' PMA. The results of network comparisons for BPD

Table 2 SUCRA ranking probabilities of corticosteroids for primary and secondary outcomes

\begin{tabular}{|c|c|c|c|}
\hline \multirow[b]{2}{*}{ Corticosteroids or placebo } & \multicolumn{3}{|l|}{ SUCRA $(\%)^{*}$} \\
\hline & $\begin{array}{l}\text { BPD at } \\
36 \text { weeks' PMA } \\
(\%)\end{array}$ & $\begin{array}{l}\text { Death at } \\
36 \text { weeks' PMA } \\
(\%)\end{array}$ & $\mathrm{CP}(\%)$ \\
\hline Dexamethasone (high dose) & 91.2 & 74.1 & 26.9 \\
\hline Dexamethasone (low dose) & 56.0 & 64.5 & 76.8 \\
\hline Budesonide & 54.9 & 23.8 & - \\
\hline Fluticasone & 78.8 & 47.1 & - \\
\hline Beclomethasone & 28.0 & - & 46.6 \\
\hline Placebo & 6.9 & 40.5 & - \\
\hline Hydrocortisone & 34.2 & - & 31.4 \\
\hline
\end{tabular}

* Larger SUCRA values denote more effective interventions.

BPD, bronchopulmonary dysplasia; CP, cerebral palsy; PMA, postmenstrual age;

SUCRA, surface under the cumulative ranking curve. 
and $\mathrm{CP}$ are shown in table 1 , and the results for other outcomes are available in online supplementary appendix $6 \mathrm{~A}$.

The use of dexamethasone, at either high or low dose, decreased the risk of BPD compared with placebo (OR 0.29, $95 \%$ CrI 0.14 to 0.52 ; OR $0.58,95 \%$ CrI 0.39 to 0.76 , respectively). High-dose dexamethasone was associated with a lower risk of BPD at 36 weeks' PMA, as compared with hydrocortisone (OR 2.53, 95\% CrI 1.20 to 5.68), beclomethasone (OR 2.75, $95 \%$ CrI 1.23 to 6.78 ) and low-dose dexamethasone (OR 1.99, 95\% CrI 1.04 to 3.91) (online supplementary appendix 6A). According to the estimated probability of being the best for BPD prevention at 36 weeks' PMA, high-dose dexamethasone ranked first (91.2\%) and beclomethasone ranked last (28.0\%) (table 2).

In terms of mortality, all corticosteroids failed to show any superiority compared with placebo, and no statistically significant difference was found between different corticosteroids (online supplementary appendix 6A).

\section{Safety: CP}

Compared with placebo, high-dose dexamethasone, hydrocortisone and beclomethasone tended to have a higher risk of CP, although the difference was statistically non-significant (ORs ranging from 1.47 to 2.02 ). No statistically significant difference was found between the five corticosteroids (online supplementary appendix 6A). According to SUCRA probabilities, low-dose dexamethasone was the first (76.8\%), ranked higher than hydrocortisone, while high-dose dexamethasone was the last (26.9\%).

Of note, two dexamethasone trials and three hydrocortisone trials were discontinued early due to safety considerations. ${ }^{29}$ 31-34 Of the five prematurely terminated trials, two were stopped due to an increased incidence of spontaneous gastrointestinal perforation in participants of the trials, ${ }^{31} 32$ while the others were terminated because of the serious adverse effects reported in other studies and in the 2002 statement from the AAP. ${ }^{313536}$

\section{Comparisons of different dexamethasone regimens}

We compared different regimens of dexamethasone, one of commonly used corticosteroids in clinical practice, to determine its optimal regimen. ${ }^{39}$

\section{Efficacy: BPD and death}

Compared with placebo, early-initiation, high-dose, long-term (EHL), and early-initiation, low-dose, long-term (ELL) dexamethasone regimens were associated with a decreased risk of BPD at 36 weeks' PMA (OR 0.11, 95\% CrI 0.02 to 0.41 ; OR $0.37,95 \%$ CrI 0.16 to 0.67 ) (table 1 ). In addition, EHL was more effective than early-initiation, low-dose, short-term (OR 7.24, 95\% CrI 1.60 to 43.15), early-initiation, high-dose, shortterm (OR 8.56, 95\% CrI 1.14 to 82.62), and late-initiation, low-dose, long-term (LLL) (OR 6.07, 95\% CrI 1.09 to 37.88). No statistically significant differences were found between different dexamethasone regimens in terms of mortality (online supplementary appendix 6B).

\section{Safety: CP}

No statistically significant differences in the risk of CP were detected between different dexamethasone regimens (online supplementary appendix 6B). According to SUCRA probabilities, high-dose and long-term regimens, late-initiation, highdose, long-term (LHL) and EHL, ranked lower than placebo in terms of CP (online supplementary appendix 7).

\section{Results of subgroup analyses}

By gestational week

For extremely preterm infants, both doses of dexamethasone and budesonide were effective in decreasing the risk of BPD at 36 weeks' PMA compared with placebo (OR 0.06, 95\% CrI 0.01 to 0.33 ; OR $0.73,95 \% \mathrm{CrI} 0.55$ to 0.97 ; OR $0.60,95 \% \mathrm{CrI}$ 0.36 to 0.93 ), even though budesonide failed to show efficacy in general preterm infants. Moreover, high-dose dexamethasone was superior to low-dose dexamethasone and budesonide in this population (OR 11.48, 95\% CrI 2.16 to 93.39; OR 9.29, $95 \%$ CrI 1.63 to 79.07$)$. No statistically significant difference was found in terms of mortality and CP between the five corticosteroids (online supplementary appendix 8).

\section{By birth weight}

For extremely low birthweight infants, only budesonide significantly reduced the risk of BPD at 36 weeks' PMA compared with placebo (OR $0.60,95 \% \mathrm{CrI} 0.34$ to 0.95 ). For very low birthweight infants, both doses of dexamethasone were associated with a decreased risk of BPD at 36 weeks' PMA (high dose: OR 0.23 , 95\% CrI 0.07 to 0.56 ; low dose: OR 0.36, 95\% CrI 0.16 to 0.70$)$. There were no statistically significant differences in the risk of CP detected between the five corticosteroids in any subgroups.

\section{DISCUSSION}

\section{Summary of findings}

Our network meta-analysis provides unified hierarchies of evidence for corticosteroid use in BPD, overcoming the problem due to inadequate evidence from head-to-head trials. Compared with placebo, both high-dose and low-dose dexamethasone reduced the risk of BPD at 36 weeks' PMA. In addition, highdose dexamethasone significantly decreased the risk of BPD compared with hydrocortisone and beclomethasone. The attempt to explore the optimal regimens of dexamethasone revealed that early initiation and a high dose of dexamethasone decreased the risk of BPD, but high-dose and long-term dexamethasone might increase the risk of CP. For the extremely preterm and extremely low birthweight infants, budesonide showed a decreased risk of BPD, compared with placebo.

\section{Hydrocortisone versus dexamethasone}

The AAP guideline discussed the difference between hydrocortisone and dexamethasone in terms of safety, based mainly on pharmacokinetic studies and animal studies. ${ }^{16}$ Our study showed that hydrocortisone ranked lower than low-dose dexamethasone in terms of both CP and BPD prevention at 36 weeks' PMA. According to a recent in vivo experimental study, hydrocortisone had similar neurotoxic effects to dexamethasone on neurons in immature chicken cerebellum and it may not be safer than dexamethasone. ${ }^{40}$

\section{Different dexamethasone regimens}

High-dose, long-term dexamethasone regimens appeared to be associated with a higher risk of CP compared with placebo (ORs range from 2.30 to 2.75) and low-dose, short-term regimens (ORs range from 2.30 to 33.33), but without significant differences. In addition, our findings appeared to support early initiation, as both EHL and ELL were associated with a lower risk of CP compared with LHL and LLL (OR: 0.82 and 0.62, respectively). The possible explanations for the finding include the following: early-initiation, low-dose dexamethasone was found to suppress the expression of inflammatory mediators 
and decrease pulmonary oedema. ${ }^{41}$ The adverse neurodevelopmental outcomes of postnatal dexamethasone therapy were shown to be associated with initiation time. Early initiation of dexamethasone enhanced the acquisition of contextual fear and spatial memory later in life of rat pups and increased dendritic spine number. ${ }^{42}$ Hence, our findings challenge the use of highdose and long-term dexamethasone, but advocate a more aggressive initiation of dexamethasone than current clinical practice.

\section{Extremely preterm and low birth weight}

In subgroup analyses, high-dose dexamethasone was shown to be superior to low-dose dexamethasone, hydrocortisone and beclomethasone in extremely preterm infants. However, the recommendation of an increase in dose for extremely preterm could not be made due to the lack of evidence on long-term neurodevelopmental safety. It was found that budesonide significantly reduced BPD at 36 weeks' PMA compared with placebo in extremely preterm and extremely low birthweight infants. This finding was consistent with the point raised by Bassler, ${ }^{43}$ who predicted that inhaled budesonide may play a role in extremely low birthweight infants. However, there was no convincing evidence from RCTs on the effects of other inhaled corticosteroids for BPD prevention. ${ }^{1144}$

\section{Limitations of this study}

Our study has several potential limitations. First, variations in corticosteroid regimens and participants resulted in significant heterogeneity across the included studies. We therefore planned a comparison of the different regimens of dexamethasone and prespecified subgroup analyses by gestational week and birth weight. Second, missing data on the exact dose and time of outcome measures reduced the number of trials included in the network meta-analysis. In addition, patient enrolment was halted early in seven of the included trials. Five trials were terminated due to adverse reactions, ${ }^{3133-36}$ while the other two were terminated because of difficulties in participant recruitment. ${ }^{30}$ Given the limited number of studies, we did not conduct sensitivity analysis on the excluded terminated trials. Finally, the effects of corticosteroids on adverse neurodevelopmental outcomes remained unclear due to the lack of trials with long-term follow-ups.

\section{CONCLUSIONS}

For preterm infants with risk of BPD, dexamethasone can reduce the risk of $\mathrm{BPD}$, and high-dose dexamethasone is more efficacious than other regimens of corticosteroids. Of the different dexamethasone regimens, aggressive initiation seems a favourable option, and a combination of high-dose and long-term use should be avoided because of the possible long-term adverse neurodevelopmental outcome. Budesonide is associated with a decreased risk of BPD in extremely preterm and extremely low birthweight infants, although recommendations cannot be made due to the lack of long-term safety data. RCTs of early-initiation and low-dose dexamethasone, budesonide and other inhaled corticosteroids with factorial design and long-term follow-ups are urgently needed to identify the optimal postnatal corticosteroid regimens.

\footnotetext{
Author affiliations

${ }^{1}$ Department of Pharmacy, West China Second University Hospital, Sichuan University, Chengdu, China

${ }^{2}$ Key Laboratory of Birth Defects and Related Diseases of Women and Children(Sichuan University), Ministry of Education, Chengdu, China ${ }^{3}$ Evidence Based Medicine Center, Lanzhou University, Lanzhou, China
}

${ }^{4}$ Key Laboratory of Evidence-based Medicine and Knowledge Translation of Gansu Province, Lanzhou University, Lanzhou, China

${ }^{5}$ Norwich Medical School, University of East Anglia, Norwich, UK

${ }^{6}$ West China School of Pharmacy, Sichuan University, Chengdu, China

${ }^{7}$ Department of Pediatrics, West China Second University Hospital, Sichuan University, Chengdu, China

${ }^{8}$ Chinese Evidence-Based Medicine Center/Chinese Cochrane Center, West China Hospital, Sichuan University, Chengdu, China

Acknowledgements We thank Professor Imti Choonara for his suggestions on this paper. We also thank the authors of the six included studies who responded to our request and provided additional data to this meta-analysis.

Contributors LZe conceived the study, drafted the study protocol, analysed the data, and drafted and revised the manuscript. JT revised the study protocol, and analysed and interpreted the data. FS contributed to the conception and design of the study, analysed and interpreted the data, and revised the manuscript. WL and $\mathrm{L}$ selected the articles, extracted data and analysed the data. GG and YZ extracted data and assessed the risk of bias of included studies. $L G$ analysed and interpreted the data, and revised the manuscript. JS contributed to the conception and design of the study, revised the study protocol and interpreted the data. XS revised the study protocol, interpreted the data and revised the manuscript. DM contributed to the conception and design of the study and revised the manuscript. LZa conceived the study, interpreted the data, and drafted and revised the study protocol and manuscript.

Funding This study was funded by the National Natural Science Foundation for Young Scholars of China (no 71503177) and the National Natural Science Foundation of China (no 81373381).

Competing interests None declared.

Provenance and peer review Not commissioned; externally peer reviewed.

Open access This is an open access article distributed in accordance with the Creative Commons Attribution Non Commercial (CC BY-NC 4.0) license, which permits others to distribute, remix, adapt, build upon this work non-commercially, and license their derivative works on different terms, provided the original work is properly cited and the use is non-commercial. See: http://creativecommons.org/ licenses/by-nc/4.0/

(C) Article author(s) (or their employer(s) unless otherwise stated in the text of the article) 2018. All rights reserved. No commercial use is permitted unless otherwise expressly granted.

\section{REFERENCES}

1 Stoll BJ, Hansen NI, Bell EF, et al. Eunice Kennedy Shriver National Institute of Child Health and Human Development Neonatal Research Network. Neonatal outcomes of extremely preterm infants from the NICHD Neonatal Research Network. Pediatrics 2010;126:443-56.

2 Walsh MC, Yao Q, Gettner P, et al. Impact of a physiologic definition on bronchopulmonary dysplasia rates. Pediatrics 2004;114:1305-11.

3 Jobe AH, Bancalari E. Bronchopulmonary dysplasia. AmJ Respir Crit CareMed 2001;163:1723-9.

4 Ambalavanan N, Walsh M, Bobashev G, et al. NICHD Neonatal Research Network. Intercenter differences in bronchopulmonary dysplasia or death among very low birth weight infants. Pediatrics 2011;127:106-16.

5 Baraldi E, Filippone M. Chronic lung disease after premature birth. N Eng/ J Med 2007:357:1946-55.

6 Doyle LW, Ehrenkranz RA, Halliday HL. Early ( $<8$ days) postnatal corticosteroids for preventing chronic lung disease in preterm infants. Cochrane Database Syst Rev 2014;5:CD001146.

7 Doyle LW, Ehrenkranz RA, Late HHL. Late (> 7 days) postnatal corticosteroids for chronic lung disease in preterm infants. Cochrane Database Syst Rev 2014;5:CD001145.

8 Halliday HL, Ehrenkranz RA, Doyle LW. Moderately early (7-14 days) postnatal corticosteroids for preventing chronic lung disease in preterm infants. Cochrane Database Syst Rev 2001;1:CD001144.

9 PRISMA. Transparent reporting of systematic reviews and meta-analyses. http:// prisma-statement.org/

10 Shah SS, Ohlsson A, Halliday HL, et al. Inhaled versus systemic corticosteroids for the treatment of chronic lung disease in ventilated very low birth weight preterm infants. Cochrane Database Syst Rev 2012;84:CD002057.

11 Shah V, Ohlsson A, Halliday HL, et al. Early administration of inhaled corticosteroids for preventing chronic lung disease in ventilated very low-birth weight preterm neonates. Cochrane Database Syst Rev 2012;5:CD001969.

12 Yoder BA, Harrison M, Clark RH. Time-related changes in steroid use and bronchopulmonary dysplasia in preterm infants. Pediatrics 2009;124:673-9.

13 Kobaly K, Schluchter M, Minich N, et al. Outcomes of extremely low birth weight $(<1$ $\mathrm{kg}$ ) and extremely low gestational age ( $<28$ weeks) infants with bronchopulmonary dysplasia: effects of practice changes in 2000 to 2003. Pediatrics 2008;121:73-81. 
14 Walsh MC, Yao Q, Horbar JD, et al. Changes in the use of postnatal steroids for bronchopulmonary dysplasia in 3 large neonatal networks. Pediatrics 2006;118:1328-35.

15 Shinwell ES, Lerner-Geva L, Lusky A, et al. Less postnatal steroids, more bronchopulmonary dysplasia: a population-based study in very low birthweight infants. Arch Dis Child Fetal Neonatal Ed 2007;92:30-3.

16 Watterberg KL. American Academy of Pediatrics. Committee on Fetus and Newborn. Policy statement--postnatal corticosteroids to prevent or treat bronchopulmonary dysplasia. Pediatrics 2010;126:800-8.

17 Nuytten A, Behal H, Duhamel A, et al. Correction: Evidence-Based Neonatal Unit Practices and Determinants of Postnatal Corticosteroid-Use in Preterm Births below 30 Weeks GA in Europe. A Population-Based Cohort Study. PLoS One 2017;12:e0172408.

18 Ward M, Sinn J. Steroid therapy for meconium aspiration syndrome in newborn infants. Cochrane Database Syst Rev 2003:4:CD003485.

19 Needelman $H$, Evans M, Roberts $H$, et al. Effects of postnatal dexamethasone exposure on the developmental outcome of premature infants. J Child Neurol 2008;23:421

20. Higgins JPT, Green S. Cochrane Handbook for Systematic Reviews of Interventions Version 5.1.0 [updated March 2011]. The Cochrane Collaboration, 2011. www. handbook.cochrane.org

21 Denjean A, Paris-Llado J, Zupan V, et al. Inhaled salbutamol and beclomethasone for preventing broncho-pulmonary dysplasia: a randomised double-blind study. Eur J Pediatr 1998;157:926-31.

22 Doyle LW, Halliday HL, Ehrenkranz RA, et al. Impact of postnatal systemic corticosteroids on mortality and cerebral palsy in preterm infants: effect modification by risk for chronic lung disease. Pediatrics 2005;115:655-61.

23 Ades AE, Welton N, Lu G. Introduction to mixed treatment comparisons. http://www. bristol.ac.uk/social-community- medicine/media/ mpes/ intro-to-mtc.pdf

24 Lu G, Ades AE. Combination of direct and indirect evidence in mixed treatment comparisons. Stat Med 2004;23:3105-24.

25 Gelman A, Rubin DB. Inference from iterative simulation using multiple sequences. Stat Sci 1992;7:457-72.

26 Salanti G, Ades AE, Ioannidis JP. Graphical methods and numerical summaries for presenting results from multiple-treatment meta-analysis: an overview and tutorial. J Clin Epidemiol 2011;64:163-71.

27 Halliday HL, Ehrenkranz RA, Late DLW. 7 days) postnatal corticosteroids for chronic lung disease in preterminfants. Cochrane Database Syst Rev 2009;1:CD001145.

28 Wang R, Lagakos SW, Ware JH, et al. Statistics in medicine--reporting of subgroup analyses in clinical trials. N Engl J Med 2007;357:2189-94.

29 Sun $X$, Ioannidis JPA, Agoritsas T, et al. How to use a subgroup analysis. JAMA 2014:311:405-11.
30 Kari MA, Heinonen K, Ikonen RS, et al. Dexamethasone treatment in preterm infants at risk for bronchopulmonary dysplasia. Arch Dis Child 1993;68:566-9.

31 Anttila E, Peltoniemi O, Haumont D, et al. Early neonatal dexamethasone treatment for prevention of bronchopulmonary dysplasia. Randomised trial and meta-analysis evaluating the duration of dexamethasone therapy. Eur J Pediatr 2005; 164:472-81.

32 Doyle LW, Davis PG, Morley CJ, et al. Low-dose dexamethasone facilitates extubation among chronically ventilator-dependent infants: a multicenter, international, randomized, controlled trial. Pediatrics 2006;117:75-83.

33 Watterberg KL, Gerdes JS, Cole CH, et al. Prophylaxis of early adrenal insufficiency to prevent bronchopulmonary dysplasia: a multicenter trial. Pediatrics 2004:114:1649-57.

34 Peltoniemi O, Kari MA, Heinonen K, et al. Pretreatment cortisol values may predict responses to hydrocortisone administration for the prevention of bronchopulmonary dysplasia in high-risk infants. J Pediatr 2005;146:632-7

35 Bonsante F, Latorre G, lacobelli S, et al. Early low-dose hydrocortisone in very preterm infants: a randomized, placebo-controlled trial. Neonatology 2007;91:217-21.

36 Malloy CA, Hilal K, Rizvi Z, et al. A Prospective, Randomized, Double-Masked Trial Comparing Low-dose to Conventional Dose Dexamethasone in Neonatal Chronic Lung Disease. Int J Pediatr Neonatol 2004;5:e10473.

37 Tapia JL, Ramírez R, Cifuentes J, et al. The effect of early dexamethasone administration on bronchopulmonary dysplasia in preterm infants with respiratory distress syndrome. J Pediatr 1998;132:48-52.

38 Walther FJ, Findlay RD, Durand M. Adrenal suppression and extubation rate after moderately early low-dose dexamethasone therapy in very preterm infants. Early Hum Dev 2003;74:37-45.

39 Truffert P, Empana JP, Bréart G, et al. Treatment strategies for bronchopulmonary dysplasia with postnatal corticosteroids in Europe: the EURAIL survey. Acta Paediatr 2003;92:948-51

40 Aden P, Goverud I, Liestøl K, et al. Low-potency glucocorticoid hydrocortisone has similar neurotoxic effects as high-potency glucocorticoid dexamethasone on neurons in the immature chicken cerebellum. Brain Res 2008;1236:39-48.

41 Huang B, Wang DX, Deng W. Protective effects of dexamethasone on early acute lung injury induced by oleic acid in rats. Int J Clin Exp Med 2014;7:4698-709.

42 Tsai KJ, Sze Cl, Lin YC, et al. A Single Postnatal Dose of Dexamethasone Enhances Memory of Rat Pups Later in Life. PLoS One 2016;11:e0165752.

43 Bassler D. Inhalation or instillation of steroids for the prevention of bronchopulmonary dysplasia. Neonatology 2015;107:358-9.

44 Onland W, Offringa M, van Kaam A. Late ( $\geq 7$ days) inhalation corticosteroids to reduce bronchopulmonary dysplasia in preterm infants. Cochrane Database Syst Rev 2012:4:CD002311. 